\title{
Heat transfer enhancement in a small pipe by spinodal decomposition of a low viscosity, liquid-liquid, strongly non-regular mixture
}

\author{
F. Di Fede, P. Poesio*, G.P. Beretta \\ Università degli Studi di Brescia, Via Branze 38, 25123 Brescia, Italy
}

\section{A R T I C L E I N F O}

\section{Article history:}

Received 16 June 2010

Received in revised form 7 October 2011

Available online 25 November 2011

\section{Keywords:}

Heat transfer enhancement

Spinodal decomposition

\begin{abstract}
A B S T R A C T
We report experimental evidence of a $20-40 \%$ enhancement of the effective heat transfer coefficient for laminar flow of a partially miscible binary liquid-liquid mixture in a small diameter horizontal tube that obtains when phase separation occurs in the tube. A mixture of acetone-hexadecane is quenched into the two-phase region so as to induce spinodal decomposition. The heat transfer rate is enhanced by selfinduced convective effects sustained by the free energy liberated during phase separation. The experimental heat transfer coefficients obtained when separation occurs are compared to the corresponding values predicted for flow of a hypothetic mixture with identical properties but undergoing separation. For such comparison, the energy balance equation must carefully take into account both the sensible heat and the excess enthalpy difference between the inlet and the outlet streams because our liquid-liquid binary mixture is a very asymmetric system with large excess enthalpies. The non-ideal mixture thermodynamic properties needed for the energy balance are obtained by an empirical procedure from the experimental data available in the literature for our mixture. The experimental setup and calculation procedure is tested by experiments performed using single-phase water flow and single-phase mixture flow (above the critical point). The convective heat transfer augmentation that results in the presence of liquid-liquid phase separation may be exploited in the cooling or heating of small scale systems where turbulent convection cannot be achieved.
\end{abstract}

(c) 2011 Elsevier Ltd. All rights reserved.

\section{Introduction}

In the current rush towards miniaturization, a key element to design smaller and smaller devices is the possibility to quickly remove heat. Current state-of-the-art cooling approaches have significant limitations, particularly for high heat flux applications. The microelectronics industry makes use of forced air cooling across optimized copper-finned heat sinks with incorporated heat pipes. Despite extensive design optimizations and the relatively large size and weight of these heat sinks, the heat fluxes that can be reached are limited. Other potential cooling methods, such as thermoelectric modules and pumped liquid-phase cooling [1,2] are inefficient and unsuitable to dissipate high heat fluxes and, hence, cannot meet the required performances. Two-phase microfluidic cooling systems have received significant attention because the latent heat during liquid-vapor phase change absorbs large fluxes. Efforts have focused primarily on boiling flows in microchannels [3]. However, in these systems liquid-vapor instabilities during phase-change lead to local dry-out, non-uniform tempera-

\footnotetext{
* Corresponding author.

E-mail address: pietro.poesio@ing.unibs.it (P. Poesio).
}

ture distributions, and significant decreases in critical heat flux [4]. Despite extensive research, there remain significant problems in the implementation of this technology. In contrast, two-phase jet impingement techniques have been investigated to a much lesser extent, despite the theoretical promise of very high heat transfer coefficients with high heat dissipation capability via thin film evaporation [5]. The implementation has comparable challenges. Previous experimental attempts have typically led to pool boiling due to chamber flooding, or liquid dry-out due to insufficient liquid supply [6]. These undesired effects significantly decrease heat transfer coefficients and heat removal rates. Another recent possibility is the use of micro-/nanoengineered surfaces, which offer new opportunities to enhance and control fluid and heat transport. Significant efforts have focused on promoting nucleation sites by incorporating surface features and roughness to improve the critical heat flux in spray cooling [7], and pool boiling [8]. Carbon nanotube forests have recently been investigated to enhance nucleate and film boiling [9]. In most of these studies, the surface features were neither sufficiently organized nor controlled. Recent progress in micro-fabrication methods has enabled the creation of more defined and sophisticated micro-/nanoscale surface structures [10]. For example, superhydrophobic surfaces using 
micro-/nanostructures have been explored to reduce drag [11] to increase heat transfer coefficients by dropwise condensation [12] and to manipulate fluids and droplet directionality $[13,14]$.

In this paper, we investigate the possibility to take advantage of liquid-liquid phase separation to enhance the heat transfer in small pipes with the final aim to design and manufacture an ultra-compact heat exchanger. This is the framework within which we have to read the recent interest on heat transfer effects during spinodal decomposition [15-17]. Spinodal decomposition is the spontaneous process whereby an unstable partially miscible liquid mixture relaxes toward a lower free energy (stable) equilibrium state. During this process, an initially homogeneous liquid solution of a given composition spontaneously changes from a single-phase unstable to a two-phase stable state consisting of two separated liquid phases, of different compositions, in mutual equilibrium. This is possible only if the overall Gibbs free energy of the two separated phases is lower than that of the initial single-phase mixture. When an initially homogeneous liquid mixture at high temperature is cooled rapidly across the coexistence curve into the twophase region, it undergoes phase segregation (demixing) either by nucleation or by spinodal decomposition. Nucleation occurs when quenching takes the system in a metastable equilibrium state: it is an activated process and a free energy barrier must be overcome in order to form critical nuclei that later grow and coalesce. Spinodal decomposition occurs spontaneously, without an energy barrier to overcome: all the concentration fluctuations are amplified regardless of their size and wavelength. If the mechanism of segregation is convection dominated, as occurs for low viscosity systems, drops move against each others under the influence of non-equilibrium capillary forces, the so-called Korteweg stresses [18]. Recently, it has been shown that this self-induced disordered bulk flow can be used to increase the heat transfer rate both in a closed configuration [15] and in small pipe flow [17].

Spinodal decomposition, associated with isotropic convective motion generated by non-equilibrium capillary forces, entails a characteristic velocity between 0.1 and $1 \mathrm{~mm} / \mathrm{s}$ [15,19]. As a result of such self-induced convective motion, the heat transfer rate is much higher than that obtained by pure heat conduction. Such heat transfer enhancement mechanism is especially desirable in systems where the small dimensions prevent the onset of turbulent transport. In comparison to vapor-liquid, partially miscible solvent systems are characterized by a very low surface tension, thus the heterogeneous separation of a low-viscosity, liquid-liquid mixture is expected to be characterized by the formation of small droplets that can be beneficially used to enhance the heat transfer rates in small diameter channels, where dry-out limits the efficiency of boiling heat transfer.

In this paper, we look at the heat transfer through a small pipe using an experimental set-up similar to the one proposed by Gat et al. [17]. Differently from the previous work, we use a two component system (acetone-hexadecane) instead of a three-component mixture. For our system, as for most practical mixtures, the heat of mixing cannot be neglected and therefore it needs to be accounted for in the energy balance equation, which we carefully derive. In addition to a new set of data (which on this topic are quite scarce), we show some qualitative differences between our results and the ones presented by Gat et al. [17]. In particular, we do not find a reduction in heat transfer effectiveness (compared to single phase flow) when a critical mixture composition is used, but we always see an increase of the effective heat transfer coefficient, in agreement with previous experimental and numerical works $[15,16]$.

The paper is organized as follows. In Section 2 we derive the energy balance equation for a liquid-liquid mixture undergoing phase decomposition through a pipe when the heat of mixing cannot be neglected. In Section 3 we review the necessary thermodynamic relations that we need to model the non-ideal behavior of our mixture and we discuss the hybrid method we adopt to correlate the available mixture data for our purposes, in view of the inadequacy of the existing models. In Section 4 we describe the experimental set-up and the validation of the procedure we use to evaluate the homogeneous flow heat transfer coefficients against which we compare our experimental data. Results are presented and discussed in Section 5. Conclusions are given in Section 6.

\section{Energy balance for a partially miscible liquid-liquid mixture flow in a pipe}

In this study, we use a liquid-liquid mixture of acetone and hexadecane. This binary system has a critical temperature $T_{c}=27^{\circ} \mathrm{C}$ and the corresponding critical molar composition is $y_{c}=0.799$ where $y=y_{a c n}$ is the mole fraction of acetone and, of course, $1-y=y_{\text {hex }}$ that of hexadecane. The reason we chose this mixture is twofold:

- it is often used as a reference for spinodal decomposition of low viscosity liquid-liquid binary mixtures because the two separated phases have the same density, ruling out the effect of gravity;

- it is a very good example of a strongly non-regular mixture, for which the heat of mixing cannot be neglected.

We consider a partially miscible binary system flowing steadily through a pipe. We indicate by 1 and 2 the two components, while the two phases are referred to as ' and ", respectively. The mole fractions are defined as $y_{1}=y=\dot{n}_{1} / \dot{n}$ and $y_{2}=1-y_{1}$. The mean molecular weight is $M=y_{1} M_{1}+y_{2} M_{2}=y M_{1}+(1-y) M_{2}$. We define the molar quality of the two-phase mixture, i.e. the mole fraction of phase ", as

$\epsilon=\frac{\dot{n}^{\prime \prime}}{\dot{n}^{\prime}+\dot{n}^{\prime \prime}}$

where $\dot{n}=\dot{n}_{1}+\dot{n}_{2}$ indicates the molar flow rates. Since $\dot{n}=\dot{n}^{\prime}+\dot{n}^{\prime \prime}$ and the phase fractions are $y^{\prime}=\dot{n}_{1}^{\prime} / \dot{n}^{\prime}$ and $y^{\prime \prime}=\dot{n}_{1}^{\prime \prime} / \dot{n}^{\prime \prime}$, we have the identity

$y=\epsilon y^{\prime \prime}+(1-\epsilon) y^{\prime}$,

from which it follows that

$\epsilon=\frac{y-y^{\prime}}{y^{\prime \prime}-y^{\prime}} \quad$ and $\quad 1-\epsilon=\frac{y^{\prime \prime}-y}{y^{\prime \prime}-y^{\prime}}$.

For a fluid volume between $z$ and $z+d z$ ( $z$ denotes the axial coordinate) in a pipe with constant cross sectional area $A$ and perimeter $P$, if we assume a one-dimensional, steady, homogeneous model, the species balance equations, $d \dot{n}_{1}=d \dot{n}_{2}=0$, imply that $d y=0, d M=0, d \dot{n}=0$, and $d \dot{m}=0$, and, therefore, the energy balance can be written as

$q^{\prime \prime} P d z=\dot{n} d h=\dot{m} d h / M$

where $q^{\prime \prime}$ is the heat flux through the side walls and we neglect axial heat flux. Under the approximation of one-dimensional and homogeneous flow, the specific molar enthalpy $h$ to enter in the balance equation can be written as

$$
\begin{aligned}
h & =\epsilon h^{\prime \prime}+(1-\epsilon) h^{\prime} \\
& =y h_{11}+(1-y) h_{22}+\frac{y-y^{\prime}}{y^{\prime \prime}-y^{\prime}} h_{\mathrm{ex}}^{\prime \prime}+\frac{y^{\prime \prime}-y}{y^{\prime \prime}-y^{\prime}} h_{\mathrm{ex}}^{\prime},
\end{aligned}
$$

where $y$ is the constant overall mole fraction, imposed by the inlet conditions, $h_{11}$ and $h_{22}$ are the pure component enthalpies, functions of $T$ and $p$, with $d h_{i i}=c_{p, i i} d T+\left[v_{i i}-T\left(\partial v_{i i} / \partial T\right)_{p}\right] d p$. So, in addition to the mean contribution of the pure components, we account for the changes in phase quality and the change in the excess 
enthalpies of the two phases. In general the excess enthalpy is a function of $T, p$, and $y$, however we will neglect the pressure dependence. To compute the excess enthalpy we need a model and we come back to this later in Section 3.

Assuming that within a given cross section, i.e., for the fluid volume between $z$ and $z+d z$, the two phases' and " are in locally mutual equilibrium, we have $T^{\prime}=T^{\prime \prime}=T, \mu_{1}^{\prime}=\mu_{1}^{\prime \prime}=\mu_{1}$, and $\mu_{2}^{\prime}=\mu_{2}^{\prime \prime}=\mu_{2}$, where $\mu$ stands for the chemical potential. Coherently with our one-dimensional model, $T$ and $\mu$ are properly defined averages over the cross section. If both phases are liquid, as in our case, the conditions of local mutual equilibrium, $\mu_{i i}^{\prime}(T, p)=\mu_{i i}^{\prime \prime}(T, p)$, imply the equality of the activity coefficients [20]

$a_{i}\left(T, p, y^{\prime}\right)=a_{i}\left(T, p, y^{\prime \prime}\right)$ for $i=1,2$.

These equations can be solved for $y^{\prime}$ and $y^{\prime \prime}$ to obtain the functions $y^{\prime}=y^{\prime}(T, p)$ and $y^{\prime \prime}=y^{\prime \prime}(T, p)$, which define the binodal curve (see Fig. 1 in the next section) and are needed to differentiate Eq. (5) to obtain the expression for $d h$ to be introduced in Eq. (4). Neglecting the pressure dependence of $h_{\mathrm{ex}}^{\prime}$ and $h_{\mathrm{ex}}^{\prime \prime}$, we can write

$d h=c_{p}(T) d T+\left[v_{\mathrm{id}}-T\left(\frac{\partial v_{\mathrm{id}}}{\partial T}\right)_{p}\right] d p$,

where

$v_{\mathrm{id}}=y v_{11}+(1-y) v_{22}$

$c_{p}(T)=c_{p}^{\mathrm{id}}+\alpha(T)+\beta(T)$,

$c_{p}^{\mathrm{id}}=y c_{p, 11}+(1-y) c_{p, 22}$,

$\alpha(T)=\epsilon\left(\frac{\partial h_{\mathrm{ex}}}{\partial T}\right)_{y, p}^{\prime \prime}+(1-\epsilon)\left(\frac{\partial h_{\mathrm{ex}}}{\partial T}\right)_{y, p}^{\prime}$,

$\beta(T)=\epsilon\left(\frac{\partial y^{\prime \prime}}{\partial T}\right)_{p}\left[\left(\frac{\partial h_{\mathrm{ex}}}{\partial y}\right)_{T, p}^{\prime \prime}-\frac{h_{\mathrm{ex}}^{\prime \prime}-h_{\mathrm{ex}}^{\prime}}{y^{\prime \prime}-y^{\prime}}\right]$

$+(1-\epsilon)\left(\frac{\partial y^{\prime}}{\partial T}\right)_{p}\left[\left(\frac{\partial h_{\mathrm{ex}}}{\partial y}\right)_{T, p}^{\prime}-\frac{h_{\mathrm{ex}}^{\prime \prime}-h_{\mathrm{ex}}^{\prime}}{y^{\prime \prime}-y^{\prime}}\right]$.

Thus, we see that the energy balance equation must be closed with equations for the dependences of $h_{\mathrm{ex}}$ on $T$ and $y$, and of $y^{\prime}$ and $y^{\prime \prime}$ on $T$.

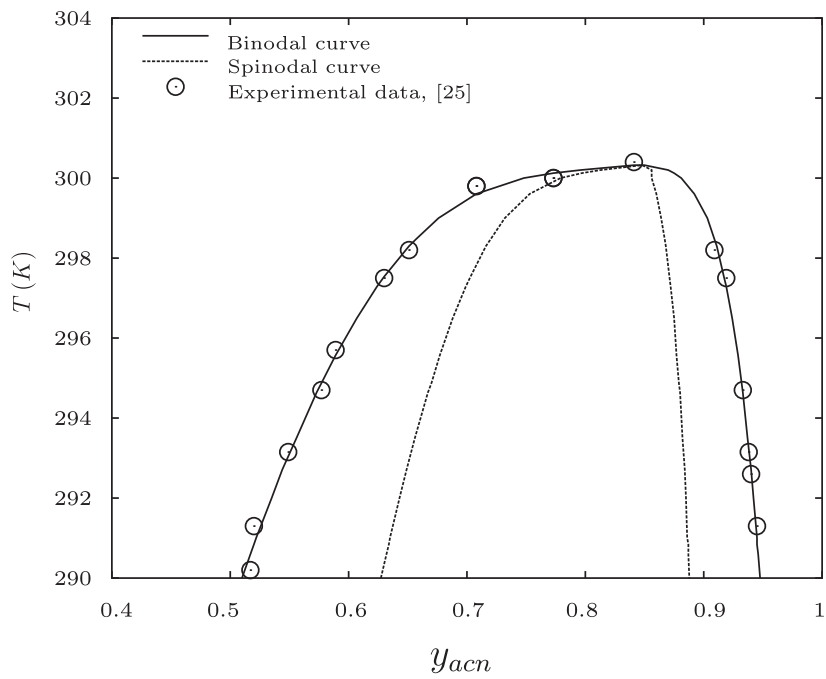

Fig. 1. Binodal and spinodal curves and experimental data from [25] for acetone in hexadecane (see text for how curves were obtained).

\section{Thermodynamically consistent estimates of non-ideal mixture properties}

For a pure substance $i, \mu_{i i}(T, p)=g_{i i}(T, p)$ and the Gibbs-Duhem relation reads as

$d g_{i i}=d \mu_{i i}=-s_{i i} d T+v_{i i} d p$,

where $g$ stands for the molar Gibbs free energy, $s$ and $v$ for the molar specific entropy and volume, respectively. The molar specific enthalpy is $h_{i i}=\left[\partial\left(\mu_{i i} / T\right) / \partial(1 / T)\right]_{p, y}$. For a mixture, within the framework of the simple system model [20], $G=\sum n_{i} \mu_{i}$ or $g=\sum y_{i} \mu_{i}, h=\sum y_{i} h_{i}$, where $h_{i}$ is the partial molar enthalpy of the constituent $i$ in the mixture defined as $h_{i}=\left[\partial\left(\mu_{i} / T\right) / \partial(1 / T)\right]_{p, y}$ and $v=\sum y_{i} v_{i}$ where the partial molar volume is $v_{i}=\left[\partial \mu_{i} / \partial p\right]_{T, y}$. In the following we focus our attention on the Gibbs free energy and the enthalpy, but similar relations can be derived for all thermodynamic properties. For a mixture, the Gibbs-Duhem relation reads

$\sum y_{i} d \mu_{i}=-\sum y_{i} s_{i} d T+\sum y_{i} v_{i} d p$

and we define the molar properties of isothermobaric mixing as

$\Delta g_{\text {mix }}=g-\sum y_{i} g_{i i}(T, p)$,

$\Delta h_{\text {mix }}=h-\sum y_{i} h_{i i}(T, p)$,

$\Delta v_{\text {mix }}=v-\sum y_{i} v_{i i}(T, p)$.

For ideal mixture behavior, $g^{\text {id }}=\sum y_{i} \mu_{i}^{\text {id }}$ with

$\mu_{i}^{\mathrm{id}}=\mu_{i i}(T, p)+R T \ln y_{i} ;$

so that $h_{i}^{\text {id }}=h_{i i}(T, p)$ and $v_{i}^{\text {id }}=v_{i i}(T, p)$, therefore, $h^{\text {id }}=\sum y_{i} h_{i i}(T, p)$ and $v^{\text {id }}=\sum y_{i} v_{i i}(T, p)$ so that $\Delta h_{\text {mix }}^{\text {id }}=0$ and $\Delta v_{\text {mix }}^{\text {id }}=0$, whereas

$\Delta g_{\text {mix }}^{\text {id }}=R T \sum y_{i} \ln y_{i}$

For a nonideal mixture, we define the excess molar properties of the mixture as $g^{\mathrm{ex}}=g-g^{\text {id }}, h^{\mathrm{ex}}=h-h^{\text {id }}$ and $v^{\mathrm{ex}}=v-v^{\text {id }}$, that combined with the previous relations, give

$\Delta g_{\text {mix }}=\Delta g_{\text {mix }}^{\text {id }}+g^{\text {ex }}$

$\Delta h_{\text {mix }}=h^{\text {ex }}$ and $\Delta v_{\text {mix }}=v^{\text {ex }}$

We also define the excess partial properties as follows

$g_{i}^{\mathrm{ex}}=\mu_{i}-g_{i i}(T, p)-R T \ln y_{i}$

$h_{i}^{\mathrm{ex}}=h_{i}-h_{i i}(T, p)$,

$v_{i}^{\mathrm{ex}}=v_{i}-v_{i i}(T, p)$.

For each constituent $i$, it is convenient to define the activity coefficient $\gamma_{i}$ (and the resulting activity $a_{i}=y_{i} \gamma_{i}$ ) by the relations

$\mu_{i}=g_{i i}(T, p)+R T \ln a_{i}=g_{i i}(T, p)+R T \ln y_{i}+R T \ln \gamma_{i}$,

so that $g_{i}^{\text {ex }}=R T \log \gamma_{i}, h_{i}^{\text {ex }}=R \partial \ln \gamma_{i} / \partial(1 / T), \quad v_{i}^{\text {ex }}=R T \partial \ln \gamma_{i} / \partial p$, and

$h^{\mathrm{ex}}=R \frac{\partial\left(g^{\mathrm{ex}} / R T\right)}{\partial(1 / T)}$

$v^{\mathrm{ex}}=R T \frac{\partial\left(g^{\mathrm{ex}} / R T\right)}{\partial p}$

Model equations such as Margules, van Laar, NRTL, UNIQUAC, UNIFAC [24], are based on some physical/kinetic assumptions and provide a formulation for the Gibbs free energy or equivalently for the activity coefficients $\gamma_{j}=\gamma_{j}\left(T, p, n_{1}, \ldots, n_{r}\right)$. Such models can be used, for example, to compute the binodal curve of a binary mixture, i.e., the expressions of $y^{\prime}=y^{\prime}(T, p)$ and $y^{\prime \prime}=y^{\prime \prime}(T, p)$, by imposing the mutual equilibrium conditions, that follow from Eq. (6) and may be written in general as 
$\Delta g_{\text {mix }}^{\prime}-\Delta g_{\text {mix }}^{\prime \prime}=\left(y^{\prime \prime}-y^{\prime}\right)\left(\frac{\partial \Delta g_{\text {mix }}}{\partial y}\right)_{T, p}^{\prime}=\left(y^{\prime}-y^{\prime \prime}\right)\left(\frac{\partial \Delta g_{\text {mix }}}{\partial y}\right)_{T, p}^{\prime \prime}$.

In general, inserting Eqs. (19) and (20) into (28) yields, after straightforward rearrangements, the following relations which define (implicitly) the expressions of $y^{\prime}=y^{\prime}(T, p)$ and $y^{\prime \prime}=y^{\prime \prime}(T, p)$, i.e., the shape of the binodal curve,

$\tilde{g}_{y}^{\mathrm{ex}}\left(T, y^{\prime}, p\right)-\tilde{g}_{y}^{\mathrm{ex}}\left(T, y^{\prime \prime}, p\right)=\ln \frac{y^{\prime \prime}\left(1-y^{\prime}\right)}{y^{\prime}\left(1-y^{\prime \prime}\right)}$

$\tilde{g}^{\mathrm{ex}}\left(T, y^{\prime}, p\right)-\tilde{g}^{\mathrm{ex}}\left(T, y^{\prime \prime}, p\right)-\left(y^{\prime}-y^{\prime \prime}\right) \frac{\tilde{g}_{y}^{\mathrm{ex}}\left(T, y^{\prime}, p\right)+\tilde{g}_{y}^{\mathrm{ex}}\left(T, y^{\prime \prime}, p\right)}{2}$

$=\frac{y^{\prime}-y^{\prime \prime}}{2} \ln \frac{y^{\prime \prime}}{y^{\prime}}+\left(1-\frac{y^{\prime}+y^{\prime \prime}}{2}\right) \ln \frac{1-y^{\prime \prime}}{1-y^{\prime}}$

where for shorthand we denoted

$\tilde{g}^{\mathrm{ex}}=\frac{g^{\mathrm{ex}}(T, y, p)}{R T}$ and $\tilde{g}_{y}^{\mathrm{ex}}=\left(\frac{\partial \tilde{g}^{\mathrm{ex}}}{\partial y}\right)_{T, p}$.

The spinodal curve for a binary mixture is determined by imposing the stability limit condition

$\left(\frac{\partial^{2} g}{\partial y^{2}}\right)_{T, p}=0$

Using Eqs. (19) and (20) into (32) yields the following relation which defines (implicitly) the expression of $T_{s}=T_{s}(y, p)$, i.e., the shape of the spinodal curve at pressure $p$

$\tilde{g}_{y y}^{\mathrm{ex}}\left(T_{s}, y, p\right)=-\frac{1}{y(1-y)}$

where of course $\tilde{g}_{y y}^{\mathrm{ex}}$ denotes $\left(\partial^{2} \tilde{g}^{\mathrm{ex}} / \partial y^{2}\right)_{T, p}$.

At the critical point for a given pressure, $y_{c}(p)=y^{\prime}\left(T_{c}, p\right)=y^{\prime \prime}\left(T_{c}, p\right)$ and Eqs. (29) and (30) become identities, moreover $\left(\partial^{2} g / \partial y^{2}\right)_{T, p}=0$ and $\left(\partial^{3} g / \partial y^{3}\right)_{T, p}=0$, therefore the function $\tilde{g}^{\mathrm{ex}}(T, y, p)$ must satisfy the conditions

$\tilde{g}_{y y}^{\mathrm{ex}}\left(T_{c}, y_{c}, p\right)=-\frac{1}{y_{c}\left(1-y_{c}\right)}$

$\tilde{g}_{y y y}^{\mathrm{ex}}\left(T_{c}, y_{c}, p\right)=\frac{1-2 y_{c}}{y_{c}^{2}\left(1-y_{c}\right)^{2}}$

where of course $\tilde{g}_{y y y}^{\mathrm{ex}}$ denotes $\left(\partial^{3} \mathrm{~g}^{\mathrm{ex}} / \partial y^{3}\right)_{T, p}$.

For our purposes it would be desirable that the available semiempirical models provide expressions for $\tilde{g}^{\mathrm{ex}}(T, y, p)$ which reproduce accurately not only the sets of available experimental data on the binodal curve but also the sets of data on the excess enthalpy and excess volume. Unfortunately, we found no such model in the literature for our mixture. In this paper however, all we need in order to interpret our experimental data is to evaluate, with good accuracy, the various terms in Eqs. (11) and (12) which are needed in the energy balance equation. Therefore, we do not address here the problem of identifying a reliable whole-purpose semi-empirical expression of $\tilde{g}^{\mathrm{ex}}(T, y, p)$ for our mixture, but it suffices that we use the most accurate suitable means to estimate the functions $y^{\prime}=y^{\prime}\left(T, p_{o}\right), y^{\prime \prime}=y^{\prime \prime}\left(T, p_{o}\right)$ and $h^{\operatorname{ex}}\left(T, y, p_{o}\right)$, where $p_{o}$ is the atmospheric pressure. Since for our conditions pressure drop is very small and the specific volumes are small for liquids, the second term in the rhs of Eq. (7) is negligible with respect to the first, and therefore we can effectively neglect pressure dependencies and omit them from now on. For $y^{\prime}=y^{\prime}(T)$ and $y^{\prime \prime}=y^{\prime \prime}(T)$ we use an empirical modification of the two-suffix Margules model, while for $h^{\mathrm{ex}}(T, y)$ we use the model proposed by Shen et Nagata [21]. We conclude this section by briefly describing such models.

In the framework of models of spinodal decomposition based on the so called $H$-model, a variation of the Van der Waals square gradient model (also known as diffuse-interface model), Vladimirova et al. [22] and Vladimirova et al. [23] used the two-suffix Margules model, $g_{i}^{\text {ex }}=R T \sum_{j} \Psi_{i j} y_{j}$ with $\Psi_{i j}=\Psi_{j i}$ and $\Psi_{i i}=0$. Mainly for its simplicity, its implementation into a numerical code is straightforward. However, the Margules model implicitly assumes that the excess Gibbs free energy, $g_{\text {ex }}=R T \sum_{i j} \Psi_{i j} y_{i} y_{j}$, is pairwise regular, i.e., symmetric with respect to exchange of $y_{i}$ with $y_{j}$. For a binary mixture

$\tilde{g}^{\mathrm{ex}}(T, y)=y_{1} y_{2} \Psi(T)=y(1-y) \Psi(T)$,

where $\Psi(T)=\Psi_{12}(T)$ is the Margules parameter.

To overcome this limitation, we consider an extension of the two-suffix Margules model based on a combination of the Redlich-Kister and the Wohl expansions [24], given by the expression

$\tilde{g}^{\mathrm{ex}}(T, y)=y(1-y)[\Psi(T)-(1-2 y) \Phi(T)]$.

We insert this expression in Eqs. (29) and (30) and obtain two relations between $y^{\prime}, y^{\prime \prime}, \Psi(T)$ and $\Phi(T)$. Using a least square procedure based on the experimental data points on $y^{\prime}(T)$ and $y^{\prime \prime}(T)$, we obtain empirical expressions of $\Psi(T)$ and $\Phi(T)$ which allow us to plot the binodal and spinodal curves shown in Fig. 1 together with the experimental data reported by Machedo and Rasmussen [25] on which they are based. From this procedure we obtain accurate estimates of the derivatives $d y^{\prime}(T) / d T$ and $d y^{\prime \prime}(T) / d T$ that are needed in Eq. (12). The resulting expressions are in excellent agreement with those obtained by a fully empirical fourth order polynomial interpolation of the experimental data. For the molar excess enthalpy, instead we could not compare our estimate with the interpolation of experimental data because these are available only at one temperature $\left(25^{\circ} \mathrm{C}\right)$, while we need to estimate $h^{\mathrm{ex}}$ as a function of $T$.

However, this model is still too simple to provide a reliable estimate of the derivatives of $h^{\mathrm{ex}}(T, y)$ that are also needed in Eqs. (11) and (12).

For that purpose, we describe the non-ideal mixture behavior by the Shen and Nagata [21] model, which is especially designed and tested for ketone-alkene mixtures. It assumes the following expression

$$
\begin{aligned}
\tilde{g}^{\mathrm{ex}}(T, y)= & y_{1} \ln \frac{\Phi_{1}^{\prime}}{y_{1}}+y_{2} \ln \frac{\Phi_{2}^{\prime}}{y_{2}}-\frac{Z}{2}\left[y_{1} q_{1} \log \frac{\theta_{1}}{\Phi_{1}}+y_{2} q_{2} \log \frac{\theta_{2}}{\Phi_{2}}\right] \\
& -\frac{Z}{2}\left[y_{1} \log \left(y_{1}+y_{2} \tau_{21}\right)+y_{1} \log \left(y_{2}+y_{1} \tau_{12}\right)\right] .
\end{aligned}
$$

where for a detailed interpretation and definition of the parameters $\Phi_{1}^{\prime}, \Phi_{2}^{\prime}, z, q_{1}, q_{2}, \theta_{1}, \theta_{2}, \tau_{12}$, and $\tau_{21}$ we refer to Appendix A (and the original paper). The corresponding excess enthalpy obtained using Eq. (26) is

$h^{\mathrm{ex}}=\frac{Z}{2} y_{1} y_{2}\left[\frac{\Delta E_{21} \tau_{21}}{y_{1}+y_{2} \tau_{21}}+\frac{\Delta E_{12} \tau_{12}}{y_{2}+y_{1} \tau_{12}}\right]$

where the parameters $\Delta E_{21}$ and $\Delta E_{12}$ are defined in Appendix $A$.

Fig. 2 shows that the model correlates the experimental data on excess enthalpy in an acceptable way. Hence, we base on this model our estimates of the derivatives $\partial h^{\mathrm{ex}} / \partial y$ and $\partial h^{\mathrm{ex}} / \partial T$ needed in Eqs. (11) and (12). On the other hand, this model provides a very poor description of the binodal curve.

The thermophysical properties of the single phase mixture are computed by the following relations. The mixture density is obtained as the mass fraction average of the densities of the pure components, $\rho=\sum x_{i} \rho_{i i}$, where the mass fractions are $x_{i}=y_{i} M_{i} / M$, and we assume that the excess volume, $v_{\mathrm{ex}}$, is negligible at all compositions. The mixture viscosity is obtained as the mole-fractionaverage of the component viscosities, $\eta=\sum y_{i} \eta_{i i}$; the empirical relations used for the viscosity and density of the two pure components as functions of temperature are reported in Appendix B. A 


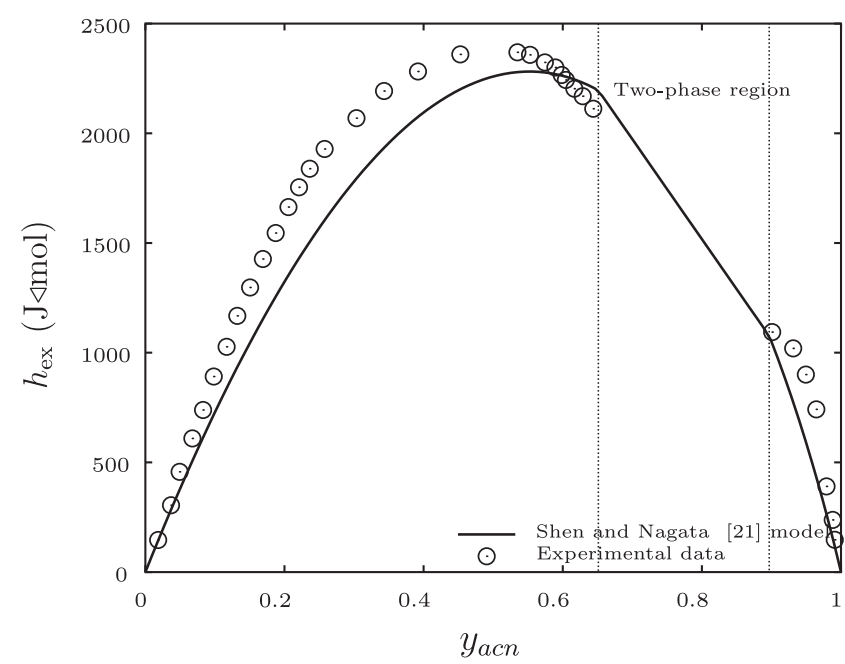

Fig. 2. Excess enthalpy $h_{e x}$ as a function of the composition $y_{\text {acn }}$ at $T=298.15 \mathrm{~K}$. Symbols represent the experimental measurements reported by Shen and Nagata [21].

more sophisticated model [26] is used for the mixture thermal conductivity of the mixture

$k=\sum \sum \psi_{i} \psi_{j} \kappa_{i j}, \quad \psi_{i}=\frac{y_{i} v_{i i}}{\sum y_{j} v_{j j}}, \quad \kappa_{i j}=\frac{2}{1 / k_{i i}+1 / k_{j j}}$,

where $v_{i i}$ is the liquid molar volume of pure component $i$. The values of the thermal conductivity as function of temperature are reported in Table B.1.

\section{Experimental set-up}

The schematic diagram of the experimental set-up used for this study, is shown in Fig. 3. It consists of a copper pipe $(D=1.7 \mathrm{~mm}$ inner diameter, $4 \mathrm{~mm}$ outer diameter, $L=180 \mathrm{~mm}$ length) placed co-axially inside a larger plastic tube (inner diameter $50 \mathrm{~mm}$ ). The acetone-hexadecane mixture is flown by means of a peristaltic pump, which imposes the volumetric flow rate. The mixture is collected at the exit of the pipe and the flow rate provided by the pump is double-checked using a scale. The mixture is kept at constant temperature in the inlet reservoir by an external thermostat and its temperature is recorded by a K-type thermocouple. All thermocouples are fabricated in our lab by wires coming from the same hank; each thermocouple is then calibrated and we could verify, using a cold bath $\left(0^{\circ} \mathrm{C}\right.$, ice/water $)$, the resulting uncertainty to be in the range $\pm 0.2{ }^{\circ} \mathrm{C}$. Before entering the inner pipe, the mixture temperature is measured by a thermocouple, $T_{\mathrm{in}}$, similarly the temperature is measured at the outlet, $T_{\text {out }}$. Cold water is passed through the external annulus. Its inlet and outlet temperatures, $T_{1}$ and $T_{2}$, are measured by two K-type thermocouples. The difference $T_{\text {in }}-T_{\text {out }}$ is recorded and analyzed to estimate the effective overall heat transfer enhancement as detailed below.

The mixture is pumped in a range of flow-rates that ensure a laminar flow, with $\operatorname{Re}=132-293$. The mixture inlet temperature

Table B.1

Thermal conductivity [W/m K] at different temperatures, Ref. [26].

\begin{tabular}{lll}
\hline $\mathrm{T}(\mathrm{K})$ & Acetone & Hexadecane \\
\hline 193.15 & 0.0198911 & 0.140 \\
289.15 & 0.190243 & 0.135 \\
303.15 & 0.17646 & 0.130 \\
348.15 & 0.168802 & 0.125 \\
\hline
\end{tabular}

$T_{\text {in }}$ and the cooling water temperature $T_{1}$ are controlled by two thermostats. The cooling water flow rate is high, so as to ensure a high external heat transfer coefficient and a very little temperature rise, as verified experimentally to a good degree of approximation. Additional thermocouples measured the wall temperature $T_{\mathrm{w}}$ along the pipe. In all cases, due to the very high flow rate of the cooling water, $T_{\mathrm{w}}$ is uniform over the entire length of the pipe. A typical experiment is carried out by setting the mixture inlet temperature, $T_{\text {in }}$, the inlet cooling temperature, $T_{1}$, and the mixture flow rate; the typical results are the mixture outlet temperature, $T_{\text {out }}$, the wall temperature, $T_{\mathrm{w}}$, and the outlet cooling water temperature, $T_{2}$.

\section{Results and discussion}

Prior to performing two-phase flow experiments, we test our experimental set-up in the simple case of a single-phase flow heat exchange through the pipe. The experimental tests consist of flowing either pure water, pure acetone, or pure hexadecane at a given flow rate with a given inlet temperature to compare the measured outlet temperature $T_{\text {out }}$ against the one predicted by the energy balance equation, $T_{\text {out }}^{\text {th }}$. To this end, Eq. (4) is complemented with $q^{\prime \prime}=U\left[T_{\mathrm{w}}-T(z)\right]$, where $T_{\mathrm{w}}$ is the constant wall temperature. Neglecting the pumping power with respect to the heat exchange rate, Eq. (7) can be written as $d h=c_{p}(T) d T$, so that we have

$\frac{\dot{m}}{M} c_{p}(T) d T=U_{\mathrm{loc}}(z)\left[T_{\mathrm{w}}-T(z)\right] \pi D d z$,

where $\pi D d z$ is an element of the effective heat exchange surface, $U_{\text {loc }}(z)$ is the local heat transfer coefficient, which (for constant fluid properties) can be written through the local Nusselt number [27] as

$\mathrm{Nu}_{\mathrm{loc}}(z)=\frac{U_{\mathrm{loc}}(z) D}{k(z)}=\frac{2}{9^{1 / 3} \Gamma\left(\frac{4}{3}\right)}\left(\mathrm{Gz}(z) \frac{L}{z}\right)^{1 / 3}$

where $\mathrm{Gz}=\operatorname{Pr} \operatorname{Re} D / L$, Pr and Re are the Graetz, Prandtl and Reynolds numbers, respectively and $\Gamma$ is the gamma function. Due to cooling, the fluid temperature changes along the pipe, $T(z)$. To account for this effect, we assume that Eq. (42) holds also for temperature varying properties and we compute $\mathrm{Pr}, \mathrm{Re}$, and $\mathrm{Gz}$ using local fluid properties evaluated at the local temperature $T(z)$; viscosity, density and specific heat are functions of temperature, estimated as detailed in Appendix B. Eq. (41) is solved numerically, together with Eq. (42), to determine the temperature distribution along the pipe and, therefore, the outlet temperature $T_{\text {out }}^{\text {homo }}$ is predicted. In Fig. 4a, the measured outlet temperatures, $T_{\text {out }}$, obtained in the pure-substance experiments are compared with the ones obtained theoretically, $T_{\text {out }}^{\text {homo }}$. As can be seen, the comparison is rather good, providing differences lower than $5 \%$, for different flow conditions (different flow rates, inlet temperature, and working fluids).

We also performed some preliminary experiments with the mixture in the single phase region, namely with the wall temperature above the critical temperature of the mixture $\left(T_{\text {out }}>T_{\mathrm{C}}=27^{\circ} \mathrm{C}\right)$. In this case, $c_{p}(T)$ is obtained as described in Section 3; the results of these experiments are reported in Fig. 4b. Also in these cases, we observe a good agreement between the measured values and the theoretical ones, although the predicted outlet temperatures are smaller than the measures one, indicating that the heat transfer coefficient resulting from our use of Eq. (42) underestimates the actual value. We attribute this small underestimate to the inaccuracy introduced by the simple relations we assumed to estimate the mixture viscosity, density, and thermal conductivity from the substance values as functions of temperature, as needed to evaluate Pr, Re, and $\mathrm{Nu}$ in Eq. (42). 


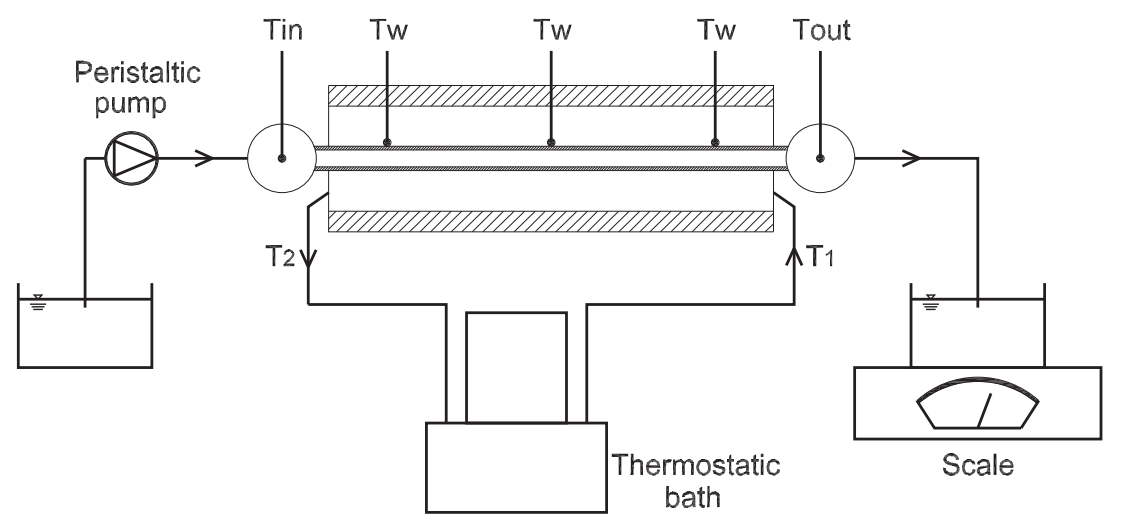

Fig. 3. Sketch of the experimental set-up.

If we define the mean heat transfer coefficient in the pipe from 0 to $L$ as $\bar{U}=\frac{1}{L} \int_{0}^{L} U_{\text {loc }}(z) d z$, with $U_{\text {loc }}$ given by Eq. (42), we can show that

$\bar{U}_{\text {homo }}=\frac{1}{D L} \int_{0}^{L} k(z) \mathrm{Nu}_{\mathrm{loc}}(z) d z=\frac{\dot{m}}{M A} \int_{T_{\text {in }}}^{T_{\text {out }}^{\text {homo }}} \frac{c_{p}(T)}{T_{\mathrm{w}}-T} d T$,

where $A=\pi D L$. Therefore, the average heat transfer coefficient can be calculated using the first integral in Eq. (43), where the local Nusselt number is obtained from Eq. (42), after the temperature distribution is obtained from the numerical solution of Eqs. (41) and (42) together with temperature dependance of the fluid properties necessary to calculate the local value of $\mathrm{Gz}(z)$. As a result, we find both the value of $T_{\text {out }}^{\text {homo }}$ and of $\bar{U}_{\text {homo }}$.

The second equality in Eq. (43) expresses an identity which follows from Eq. (41). It establishes a relation between $\bar{U}_{\text {homo }}$ and $T_{\text {out }}^{\text {homo }}$ which is independent of how $T$ varies with $z$ and depends only on how $c_{p}$ varies with $T$. For example, when $c_{p}$ can be assumed to be a constant, such relation is given by the usual expression

$\bar{U}_{\text {homo }}=\frac{\dot{m} c_{p}}{M A} \ln \left(\frac{T_{\mathrm{w}}-T_{\text {out }}^{\text {homo }}}{T_{\mathrm{w}}-T_{\text {in }}}\right)$.

We now analyze our two sets of experimental data ${ }^{1}$ obtained under spinodal decomposition conditions of an off-critical composition $\left(y_{\mathrm{acn}}=0.727\right)$ and the critical composition $\left(y_{\mathrm{acn}}=y_{\mathrm{c}}=0.799\right)$ of our acetone-hexadecane mixture. In Figs. 5 and 6, we compare the measured outlet temperature $T_{\text {out }}^{\text {meas }}$ to the theoretical value $T_{\text {out }}^{\text {homo }}$ (obtained as above by assuming the heat transfer correlation (42) valid for homogeneous flow, both in case for the critical and off-critical compositions). The experimental data are plotted as a function of the dimensionless inlet temperature $\widetilde{T}_{i}=\left(T_{i}-T_{\text {out }}^{\text {meas }}\right) /\left(T_{c}-T_{\mathrm{w}}\right)$.

The measured values of the outlet temperature $T_{\text {out }}^{\text {meas }}$ are lower than $T_{\text {out }}^{\text {homo }}$. This, we claim, is because the flow is no longer single phase and the decomposition occurs inducing the local convective enhancement described in the introduction. To support our claim, we compute the value $\bar{U}_{\text {eff }}$ that the overall heat transfer coefficient should have in homogeneous flow to yield the measured outlet temperature. Thus, based on Eq. (41), we define $\bar{U}_{\text {eff }}$ by the relation

$\bar{U}_{\text {eff }}=\frac{\dot{m}}{M A} \int_{T_{\text {in }}}^{T_{\text {out }}^{\text {meas }}} \frac{c_{p}(T)}{T_{\mathrm{w}}-T} d T$,

and we define the Augmentation Factor, i.e. the increase of the heat transfer coefficient due to the spinodal induced convection, as

\footnotetext{
${ }^{1}$ The data used in this paper are made available in the form of an Excel file and they can be requested either to the Editor or directly to the Authors.
}

$A F=\frac{\bar{U}_{\text {eff }}-\bar{U}_{\text {homo }}}{\bar{U}_{\text {homo }}}=\frac{\int_{T_{\text {out }}}^{T_{\text {out }}^{\text {meas }}} \frac{c_{p}(T)}{T_{w}-T} d T}{\int_{T_{\text {in }}}^{\text {thoum }} \frac{c_{p}(T)}{T_{w}-T} d T}$.

By such a definition we can highlight the enhancement effect produced by the convective motion induced by spinodal decomposition: we take the difference between the actual heat transfer coefficient $\left(\bar{U}_{\text {eff }}\right)$ and the one $\left(\bar{U}_{\text {homo }}\right)$ that we would obtain for laminar flow (with no spinodal induced microagitation) of a homogeneous fluid with the same effective local values of physical properties as the experimental mixture. Such a difference is then made dimensionless. $\bar{U}_{\text {homo }}$ is the average heat transfer coefficient computed for a homogeneous fluid with the same physical properties as the mixture used in the experiments (in particular, with the same specific heat, i.e., $\left.c_{p}(T)=c_{p}^{\text {id }}+\alpha(T)+\beta(T)\right)$; since the computed value of $T_{\text {out }}^{\text {homo }}$ may be larger than the critical temperature $\left(T_{c}\right)$ while the actual measured temperature may be smaller, we used the same expression for the specific heat, but we computed it at the corresponding temperature, and therefore at $T>T_{c}$ we used $\alpha(T)=0$ and $\beta(T)=0$. The values of $\alpha(T)=0$ and $\beta(T)=0$ inside the curve are computed by means of Eqs. (11) and (12) and are plotted in Fig. 7 for the critical composition mixture. If the measured outlet temperature is equal to the one of the homogeneous case (i.e. $T_{\text {out }}^{\text {meas }}=T_{\text {out }}^{\text {homo }}$ ), there is no increase in the heat transfer coefficient; on the contrary, if $T_{\text {out }}^{\text {meas }}<T_{\text {out }}^{\text {homo }}$, an increase due to spinodal induced convection is present. The only difference between the experimental mixture and the hypothetical homogeneous fluid used in the theoretical computation of $\bar{U}_{\text {homo }}$ is that the correlation (Eq. (42)) used for the latter excludes the effects of micro-agitation induced by spinodal decomposition. The way we have defined $A F$ is actually very useful to single out the effect of decomposition-induced heat transfer enhancement. The computed values of the $A F$ are reported in Figs. 8 and 9 for the critical and off-critical composition, respectively.

If we assume a negligible excess enthalpy contribution so that $c_{p}(T)=c_{p}^{\text {id }}$ (which is justified in our cases due to the limited temperature variation), and moreover assume that $c_{p}^{\text {id }}$, we obtain an ideal Augmentation Factor, $A F_{\text {id }}$, which be Eq. (47) is given by

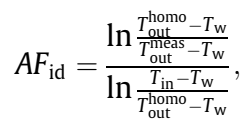

where the last approximation holds when, like in our case, $\left|T_{\text {out }}^{\text {homo }}-T_{\text {out }}^{\text {meas }}\right| \ll\left|T_{\text {out }}^{\text {homo }}-T_{\mathrm{w}}\right|$. The augmentation factor $A F_{\text {id }}$ is closely related to that adopted by Gat et al. [17], defined as $A F_{\mathrm{Gat}}=\bar{U}_{\text {eff }} / \bar{U}_{\text {homo, }}$, i.e. $A F_{\text {id }}=A F_{\text {Gat }}-1$. We can therefore introduce

$\psi=\frac{\left(A F_{\text {id }}-A F\right)}{A F}$, 


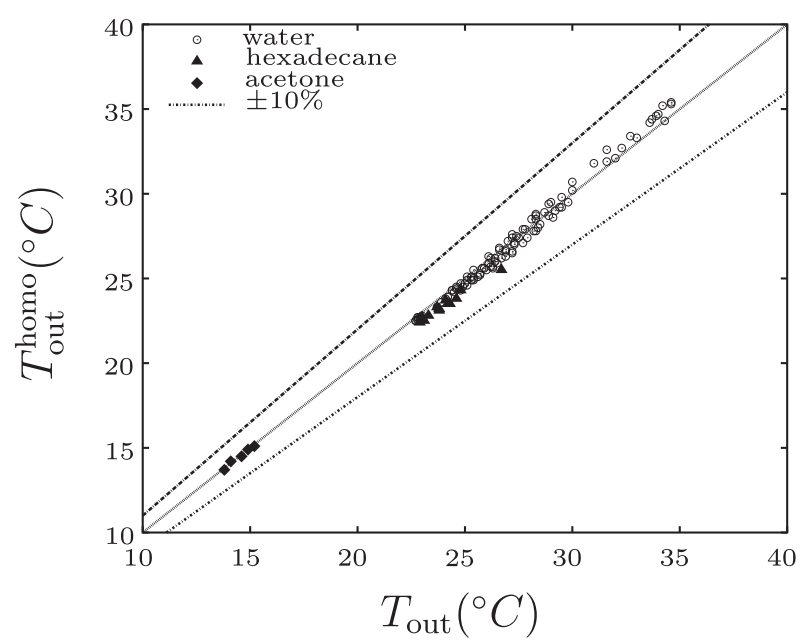

(a) Comparison between the measured outlet temperature and the one predicted by Eq. 43 for pure substances (water, acetone, and hexadecane). Temperature uncertainty is $\pm 0 \triangleright 2^{\circ} \mathrm{C}$.

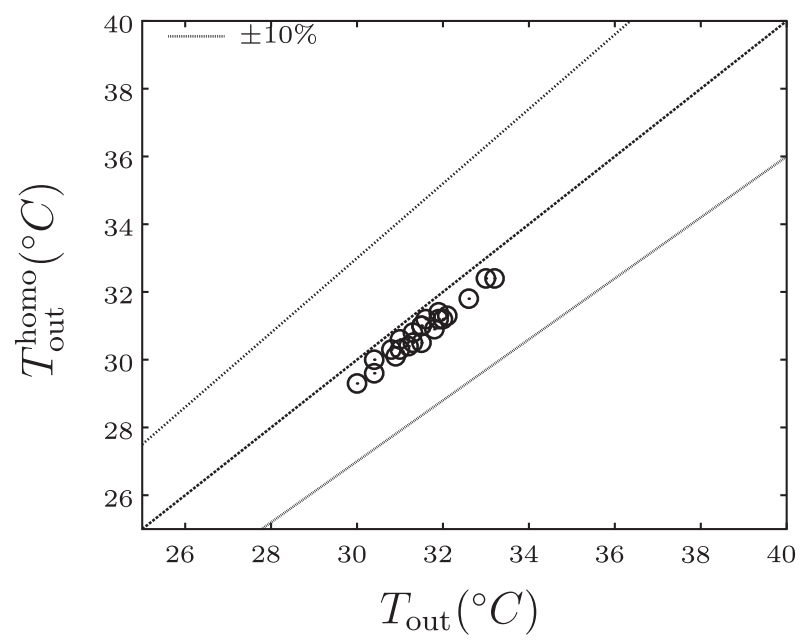

(b) Comparison between the measured outlet temperature and the one predicted by Eq. 43 with $c_{p}(T)$ given (see text) by Eqs. 9-12 for single phase flow of mixtures of different compositions of acetone and hexadecane mixture $\left(T_{\text {in }}>T_{\text {out }}>T_{\mathrm{c}}\right)$. Temperature uncertainty is $\pm 0 \triangleright 2^{\circ} \mathrm{C}$.

Fig. 4. Comparison between the measured outlet temperature and the one predicted by Eq. (43).

which represents the error made in the estimate of $A F$ by neglecting the excess enthalpy contributions in the energy balance, see Fig. 10.

For off-critical mixture composition, our results agree with the previous work by Gat et al. [17]. The experimental outlet temperature $T_{\text {out }}^{\text {meas }}$ is smaller than would be predicted by correlations for homogeneous flow, indicating a more efficient heat transfer which we believe is induced by the vigorous local mixing effect caused by spinodal decomposition through Korteweg forces. Fig. 9 shows that

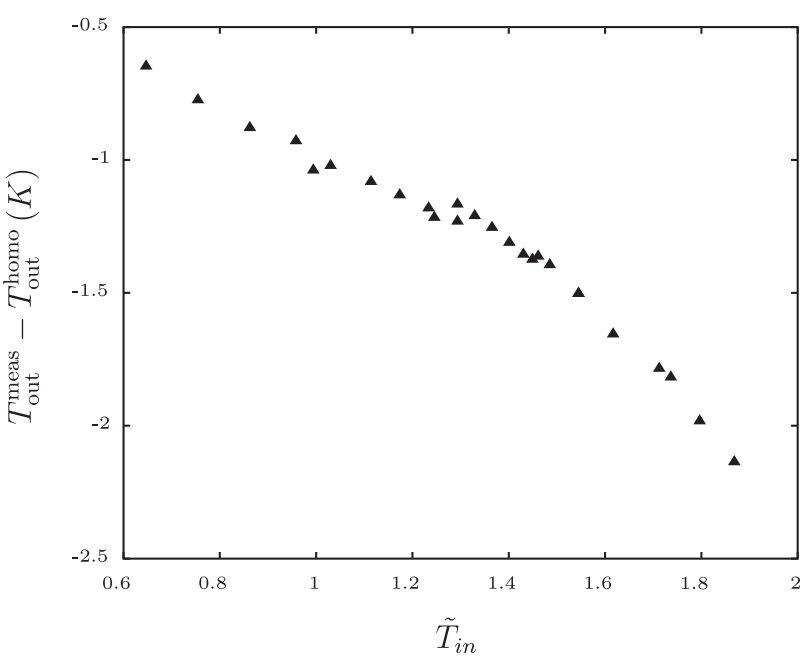

Fig. 5. Difference $T_{\text {out }}^{\text {meas }}-T_{\text {out }}^{\text {homo }}$ as a function of the dimensionless inlet temperature $\widetilde{T}_{\text {in }}=\left(T_{\text {in }}-T_{\text {out }}^{\text {meas }}\right) /\left(T_{\mathrm{c}}-T_{\mathrm{w}}\right)$ for the off-critical composition $\left(y_{\mathrm{acn}}=0.727\right)$.

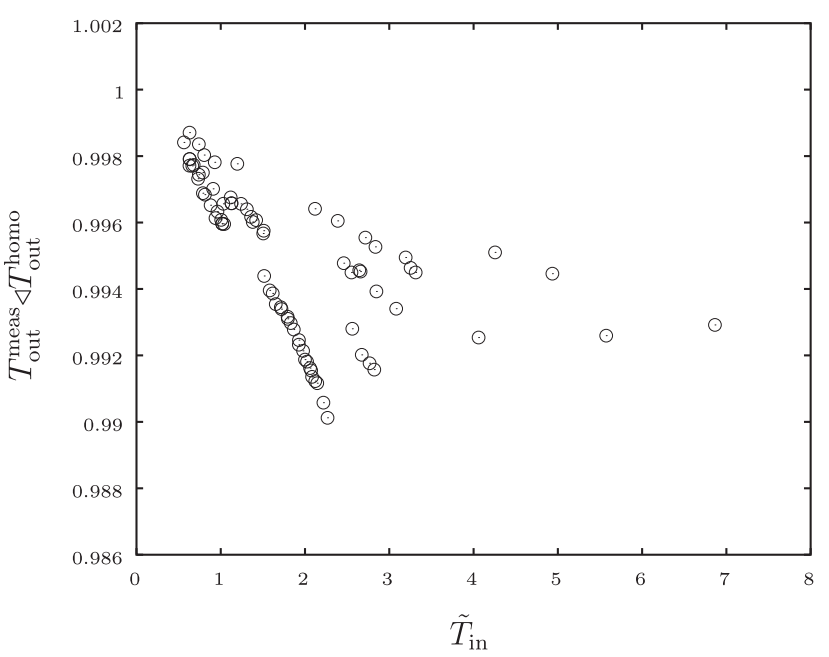

Fig. 6. Ratio $T_{\text {out }}^{\text {meas }} / T_{\text {out }}^{\text {homo }}$ as a function of the dimensionless inlet temperature $\widetilde{T}_{\text {in }}=\left(T_{\text {in }}-T_{\text {out }}^{\text {meas }}\right) /\left(T_{\mathrm{c}}-T_{\mathrm{w}}\right)$ for the critical composition $\left(y_{\mathrm{c}}=0.799\right)$.

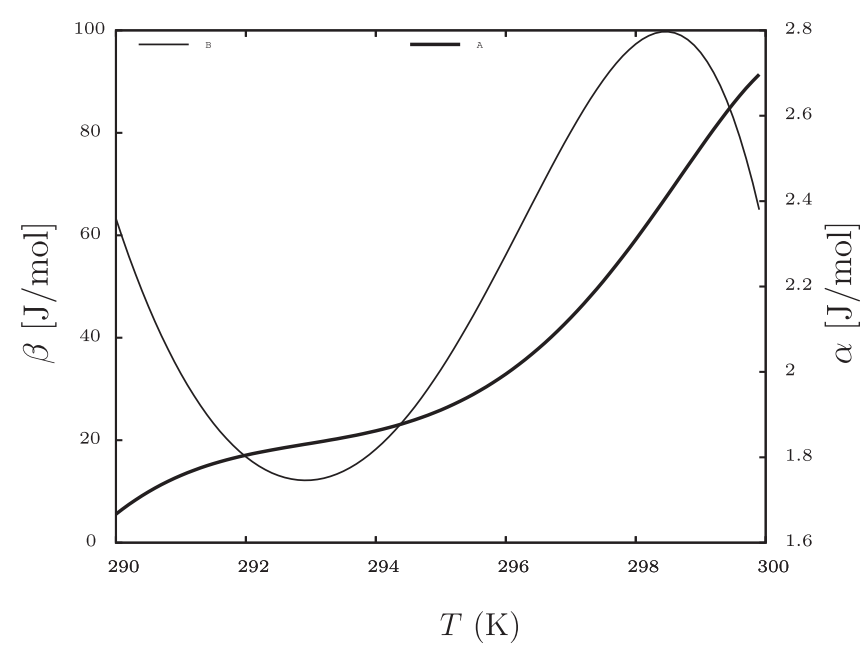

Fig. 7. $\alpha(T)=0$ and $\beta(T)=0$ for the critical mixture acetone-hexadecane, at $T<T_{c}$, as a function of temperature. 


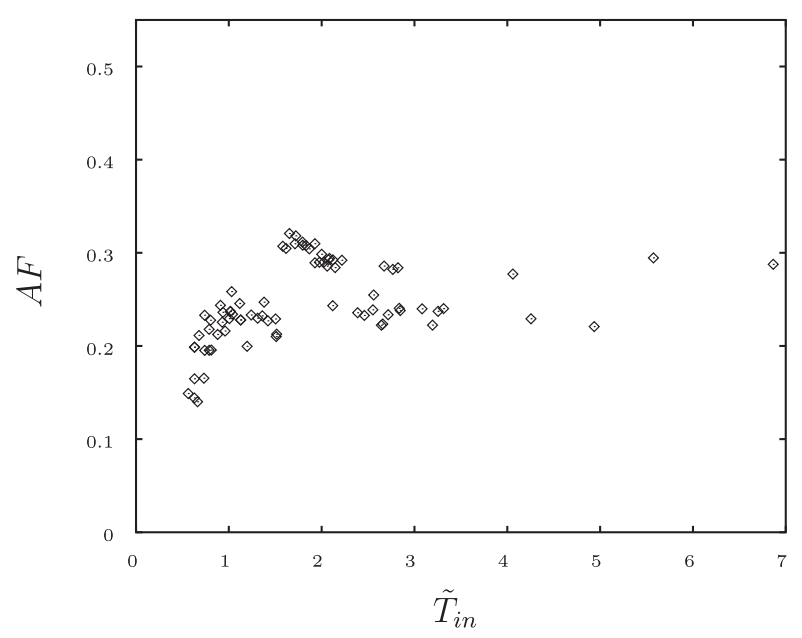

Fig. 8. Measured $A F$ as a function of the dimensionless inlet temperature $\widetilde{T}_{\text {in }}=\left(T_{\text {in }}-T_{\text {out }}^{\text {meas }}\right) /\left(T_{\mathrm{c}}-T_{\mathrm{w}}\right)$ for the critical composition $\left(y_{\text {acn }}=0.799\right)$.

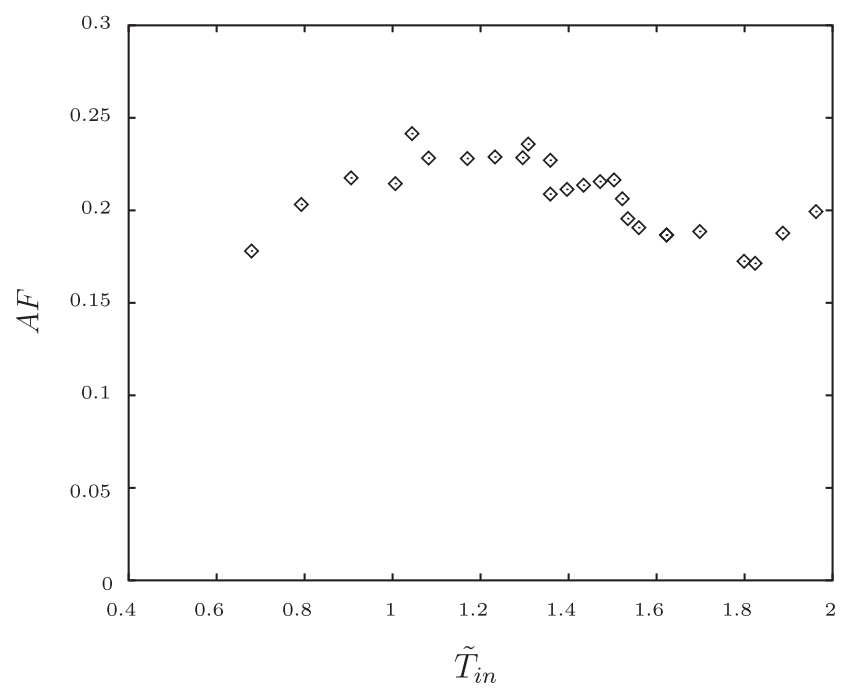

Fig. 9. Augmentation factor $A F$ as a function of the dimensionless inlet temperature $\widetilde{T}_{\text {in }}=\left(T_{\text {in }}-T_{\text {out }}^{\text {meas }}\right) /\left(T_{\mathrm{c}}-T_{\mathrm{w}}\right)$ for the off-critical composition $\left(y_{\text {act }}=0.727\right)$.

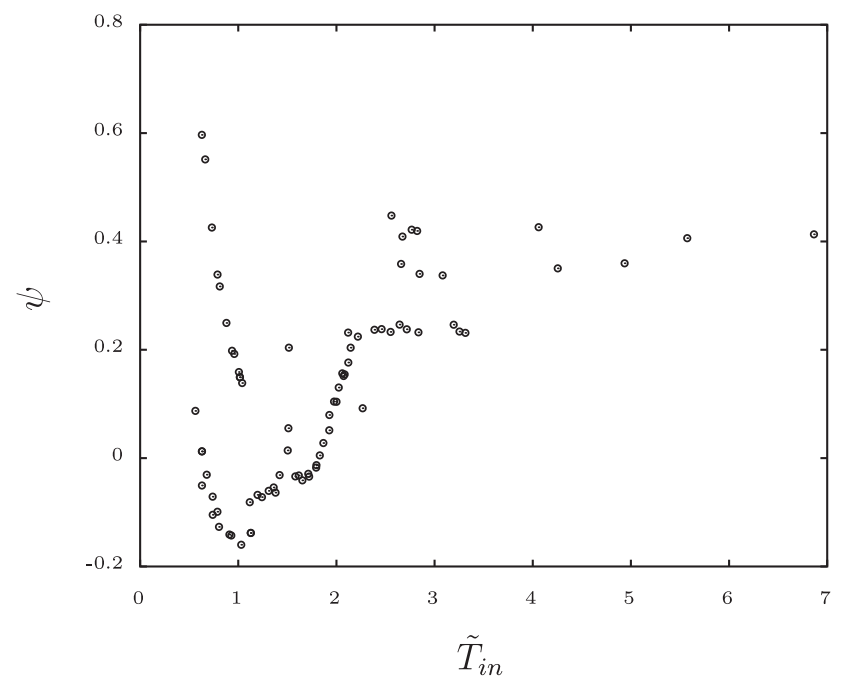

Fig. 10. $\psi=\left(A F_{\text {id }}-A F\right) / A F$ as a function of the temperature for the critical mixture composition. the effective heat transfer coefficient is up to $20 \%$ larger than for homogeneous flow. Based on their data, Gat et al. [17] proposed the following empirical correlation for the augmentation factor

$A F_{\mathrm{Gat}}=0.111\left(\frac{T_{\mathrm{in}}-T_{\mathrm{out}}^{\text {meas }}}{T_{\mathrm{c}}-T_{\mathrm{w}}} \frac{D}{L} \mathrm{Pe}\right)^{0.75} \exp \left[1.9\left(\frac{T_{\mathrm{c}}-T_{\text {out }}^{\text {meas }}}{T_{\mathrm{c}}-T_{\mathrm{w}}}\right)^{3.6}\right]$,

where Pe is the mass Péclet number. Fig. 11 compares the measured effective heat transfer coefficient $\bar{U}_{\text {eff }}$ with the "predicted" value $\bar{U}_{\text {Gat }}=\bar{U}_{\text {homo }} A F_{\text {Gat }}$, where $\bar{U}_{\text {homo }}$ is computed from the single phase correlation and $A F_{\mathrm{Gat}}$ is computed from Eq. (49). It shows that the agreement is rather poor and we conclude that the validity of Eq. (49) is appears quite limited. In Appendix C further comparisons between our results and the one predicted by the Gat et al. [17] correlation are provided.

Fig. 6 shows the ratio between the actual outlet temperature and the corresponding theoretical value for homogeneous flow as function of $\widetilde{T}_{\text {in }}$, obtained using a mixture with critical composition. Also in this case we find that the actual outlet temperature is always lower than the homogeneous value, indicating a more efficient heat transfer mechanism. Furthermore, if we compare $T_{\text {out }}^{\text {meas }}$ and $T_{\text {out }}^{\text {homo }}$ in Fig. 12, we identify two regions. Region (A) corresponds to conditions for which phase separation takes place inside the test section; the mixture enters the test section above the critical temperature and exits below the critical temperature $T_{\text {in }}>T_{\mathrm{c}}, T_{\text {out }}^{\text {meas }}<T_{\mathrm{c}}$. The mechanism of spinodal segregation produces a self-induced local stirring, which contributes to improve the heat transfer. In region (B), the mixture exits the test section with a bulk temperature higher than its critical value $\left(T_{\text {out }}^{\text {meas }}>T_{\mathrm{c}}\right)$, but the wall temperature $T_{\mathrm{w}}$ is maintained well below $T_{\mathrm{c}}$. Therefore, near the wall the mixture temperature is below $T_{\mathrm{c}}$ and the mixture phase-separates. Thus, the selfinduced local stirring caused by spinodal phase separation is confined to the near-wall layers and results in a more effective convective heat transfer. Similar conclusions can be drawn from the computation of the effective heat transfer coefficient $\bar{U}_{\text {eff }}$ and the corresponding augmentation factor. In the case of the critical mixture, we see an increase of the effective heat transfer coefficient up to $40 \%$, see Fig. 12 .

For the critical mixture composition, our results are in contrast with those of Gat et al. [17] who reported a reduction in heat transfer efficiencies during spinodal decomposition and attributed this effect to dendritic morphology which inhibits convection. Our experiments show an increase in $\bar{U}$ even when the composition

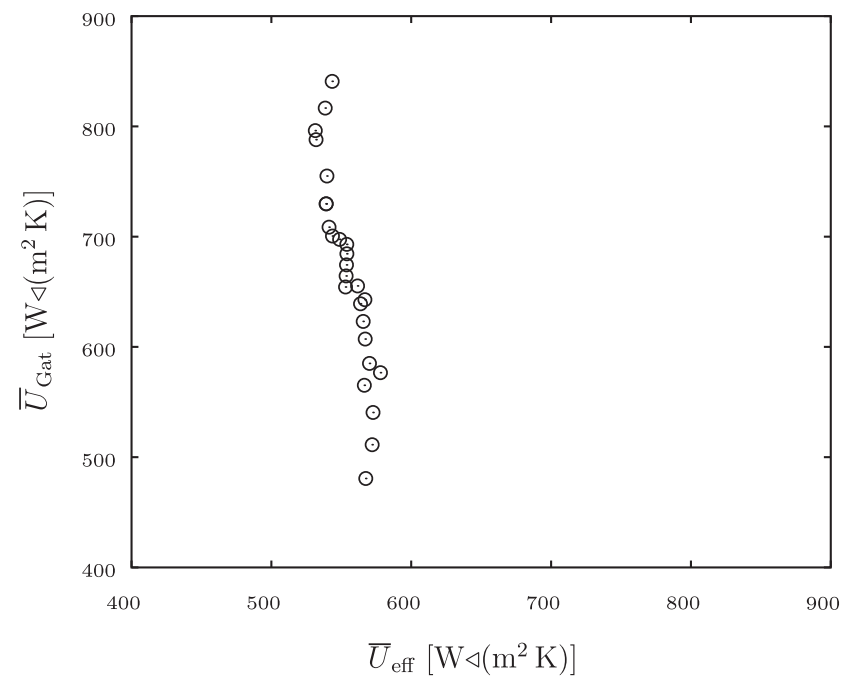

Fig. 11. Comparison between the measured $\bar{U}_{\text {eff }}$ and the $\bar{U}_{\text {Gat }}$ predicted by Eq. (49) for the off-critical composition $\left(y_{\text {act }}=0.727\right)$. 


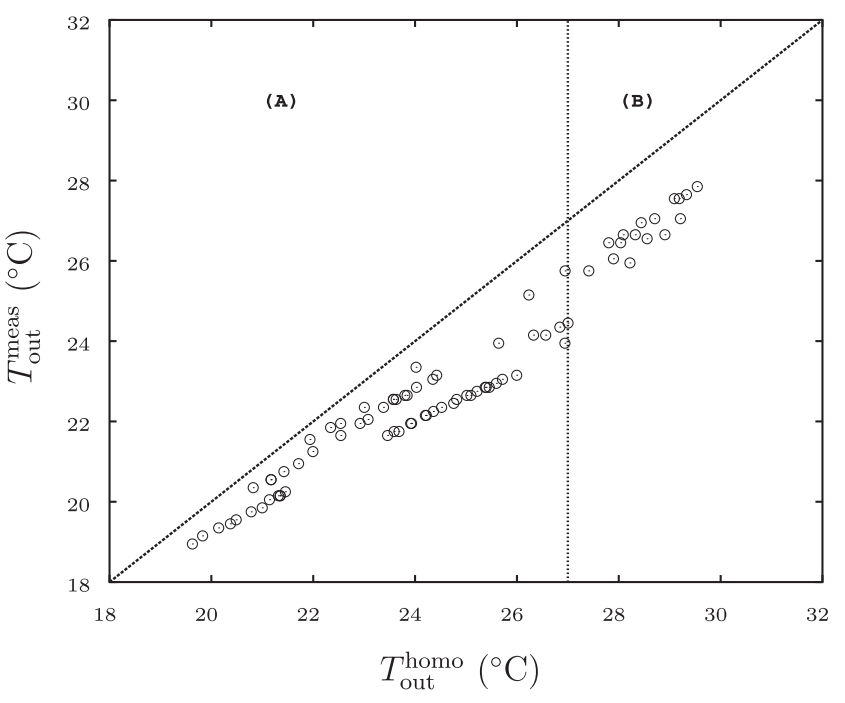

Fig. 12. Comparison between $T_{\text {out }}^{\text {homo }}$ and $T_{\text {out }}^{\text {meas }}$.

is critical. The reason for such difference in the two otherwise very similar experiments remains unclear, but most likely it is due to the difference in the mixture used. It is worth mentioning however that even for the critical composition mixture we have not seen (using an experimental set-up not described in the paper) a dendritic structure, contrary to what we have seen in our previous work [28]. There may be several reasons for that:

- we used a different mixture with different thermo-physical properties and our quenching rate is rather small, $3^{\circ} \mathrm{C} / \mathrm{min}$, compared to the $5-7^{\circ} \mathrm{C} / \mathrm{s}$ normally used in rapid quenching experiments; therefore even if the overall composition is critical, after an initial stage we have the formation of two off-critical mixtures that separate in a drop-wise morphology;

- the decomposition occurs under shear, which may cause elongation and fracture of the dendritic structures into droplets;

- within the pipe there is a radial thermal gradient inducing Marangoni flows that can further split the dendritic structure into small droplets.

The enhancement found in our flow experiments, about 20$40 \%$, is less dramatic than the four to sixfold decrease in cooling time we found in static mini-vessel $(0.1 \mathrm{~mm}$ thickness) experiments [15]. This is probably due to the much smaller quenching rates that we could achieve in our relatively large $(1.7 \mathrm{~mm}$ i.d.) diameter pipe.

\section{Conclusions}

In this paper, we presented experimental results on the effects of spinodal decomposition on flow heat transfer in a small pipe for a low viscosity liquid-liquid mixture (acetone-hexadecane) which presents a lack of miscibility at low temperatures. Since spinodal decomposition of low viscosity mixtures is known to be characterized by vigorous self-induced stirring due to Korteweg capillary forces, we show experimentally that the effective heat transfer is enhanced compared to homogeneous flow conditions. Such an enhancement is reported by the definition of the Augmentation Factor $A F$, which gives an idea of the effect of the convective motion on the heat transfer. Similarly to what proposed by [17], $A F$ can used as a correction coefficient, similarly as it is done in normal practice during the design of heat exchanger with complex geometry, first the relation (43) can be used to compute the heat transfer coefficient in case of a homogeneous mixture with the same phys- ical properties and then this value can be corrected by the $A F$. So far, only few relations for the $A F$ are present in the literature-the one presented here and the one proposed by [17]-but we have hope that in the future, encouraged by the evidences that spinodal mixture can provide a valid solution for heat transfer enhancement, new and more detailed relations will arise.

The estimation of the AF is conservative, i.e. we expect to have an higher AF than the one reported here. As can be seen from the Fig. $4 \mathrm{~b}$ the outlet temperature for the single-phase mixture is consistently under-predicted (probably because of the relations used to compute the mixture properties) and, therefore, we are consistently over-predicting the single phase heat transfer coefficient, resulting in a smaller $A F$. We have decided to not correct the output of the calculation obtained by Eq. (43) to match the experimental results, but we can evaluate that, roughly, the AF is about $15 \%$ larger than the value reported in the paper.

In contrast with recent data presented by Gat et al. [17] that show an increase of heat transfer efficiency for off-critical composition and a decrease for critical composition, our experimental data demonstrate a sizable heat transfer enhancement effect (20-40\%) for both off-critical and critical mixture compositions.

\section{Appendix A. Parameters to be used in the Shen and Nagata [21] model}

If we indicates with $v_{i}$ the saturated volume at $293.15 \mathrm{~K}, q_{i}$ the molecular surface area parameter, $r_{i}$ the molecular volume parameter of the $i$ component, the parameters entering in the Shen and Nagata [21] model can be defined as

$$
\begin{aligned}
v_{i j} & =\frac{v_{i}+v_{j}}{2} \\
\tau_{i j} & =\frac{v_{i j}}{v_{j}} \exp \left(-\alpha \Delta E_{i j} / R T\right) \\
\Delta E_{i j} & =N_{0}\left(\epsilon_{j j}-\epsilon_{i j}\right) \\
\theta_{i} & =\frac{x_{i} q_{i}}{\sum x_{k} q_{k}} \\
\phi_{i} & =\frac{x_{i} r_{i}}{\sum x_{k} r_{k}} \\
\phi_{i}^{\prime} & =\frac{x_{i} q_{i}}{\sum x_{k} q_{k}} \\
\phi_{i}^{\prime} & =\frac{x_{i} r_{i}^{3 / 4}}{\sum x_{k} r_{k}^{3 / 4}}
\end{aligned}
$$

where $N_{0}$ is Avogadro's constant; $v_{j}, q_{j}$ and $r_{j}$ are the saturated liquid volume at $293.15 \mathrm{~K}$, the molecular surface area parameter and the molecular volume parameter of component $j$, respectively. $\epsilon_{i j}$ denotes the minimum potential energy which characterizes the molecular interaction between a central molecule $i$ and a surrounding molecule $j$, while the parameter $\alpha$ is used to characterize the tendency of the components to mix in a non-random way.

\section{Appendix B. Expression for the temperature dependent fluid properties}

The following empirical relations are used to estimate the density, specific heat, and viscosity as function of temperature for the acetone and for the hexadecane, see Ref. [26].

Acetone:

density $\left[\mathrm{kmol} / \mathrm{m}^{3}\right]$ :

$\rho(T)=\frac{1.2332}{0.25866^{\left[1+\left(1-\frac{T}{508.2}\right)^{0.2913}\right]}} ;$ 


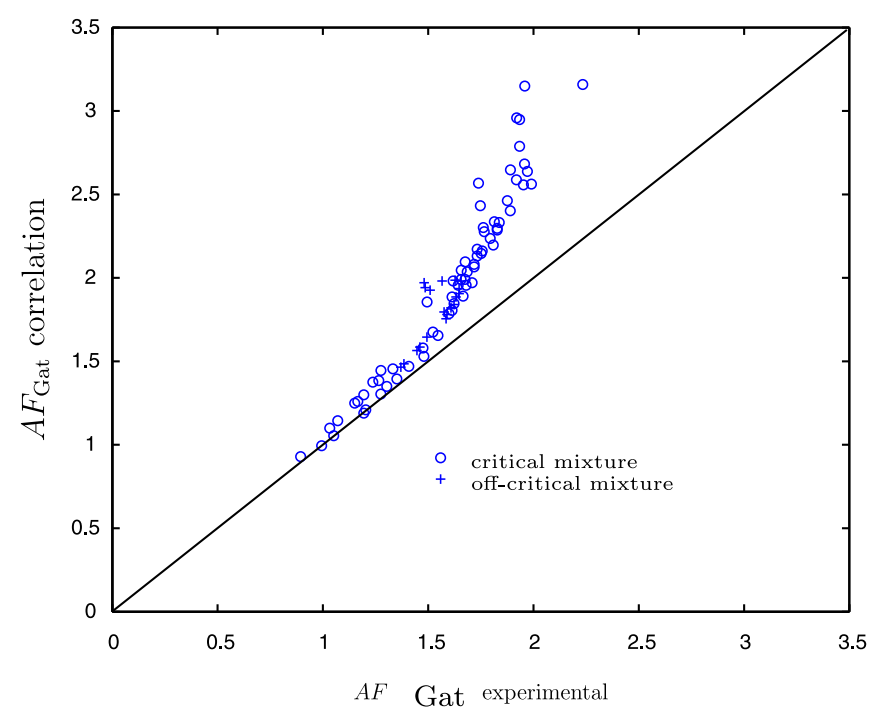

Fig. C.13. Comparison between the Augmentation Factor proposed by Gat et al. [17] obtained by our measurement $-A F_{\mathrm{Gat}}$ experimental - and the one predicted by the empirical correlation $-A F_{\text {Gat }}$ correlation - Eq. (49).

specific heat $[\mathrm{J} / \mathrm{kmol} \mathrm{K]}$ :

$c_{p}(T)=1.356 \cdot 10^{5}-1.77 \cdot 10^{2} \cdot T+0.2837 \cdot T^{2}+6.89 \cdot 10^{-4} \cdot T^{3} ;$

Viscosity [mPa s]:

$\mu(T)=\exp \left(-3.37955+\frac{553.4}{-46.97+T}\right)$.

Hexadecane:

density $\left[\mathrm{kmol} / \mathrm{m}^{3}\right]$ :

$\rho(T)=\frac{0.2687}{0.25287^{\left[1+\left(1-\frac{T}{T 23}\right)^{0.31143}\right]} ;}$

specific heat $[\mathrm{J} / \mathrm{kmol} \mathrm{K]}$ :

$c_{p}(T)=3.7035 \cdot 10^{5}-2.3147 \cdot 10^{2} \cdot T+0.68362 \cdot T^{2} ;$

Viscosity [mPa s]:

$\mu(T)=\exp \left(-4.0453+\frac{1247.3}{-55.86+T}\right)$.

The thermal conductivity is tabulated in Table B.1 and a linear interpolation between values is used.

\section{Appendix C. Comparison of the measured $A F$ and the correlation by Gat et al. [17]}

The $A F_{G a t}$ obtained by our experimental measurements can be compared to the correlation proposed by Gat et al. [17] - Eq. (49) - see Fig. C.13. In some range (corresponding to large $T_{i n}-T_{c}$ ) the correlation over-predicts the experimental $A F_{G a t}$, still it reasonably represents the experimental $A F_{G a t}$. It is worth mentioning that Gat et al. [17] experiments were conducted with $T_{\text {in }}$ close to $T_{c}$, therefore the correlation is expected to be less reliable for large $T_{\text {in }}-T_{c}$, which characterizes most of the data points presented in our work. It is worth pointing out, however, that while Gat et al. [17] definition of $A F_{G a t}$ is the same as the one presented here, their
$T_{\text {homo, }}$ and therefore their $\bar{U}_{\text {homo, }}$, is calculated based on ideal properties. We believe that such definition does not allow distinguishing between the non-ideal behavior of the mixture, which would be present also for a nonseparating mixture, and the enhancement related to the convective motion, which are peculiar of a spinodal separating mixture.

\section{References}

[1] C.T. Wang, T.-S. Leu, T.-M. Lai, Micro-capillary pumped loop system for a cooling high power device, Exp. Thermal Fluid Sci. 32 (5) (2008) 1090-1095.

[2] P. Naphon, S. Wiriyasart, Liquid cooling in the mini-rectangular fin heat sink with and without thermoelectric for CPU, Int. Comm. Heat Mass Transf. $36(2)$ (2009) 166-171.

[3] L. Zhang, E.N. Wang, K.E. Goodson, T.W. Kenny, Phase change phenomena in silicon microchannels, Int. J. Heat Mass Transf. 48 (8) (2005) 1572-1582.

[4] S.G. Kandlikar, Fundamental issues related to flow boiling in minichannels and microchannels, Exp. Thermal Fluid Sci. 26 (2002) 389-407.

[5] E.N. Wang, L. Zhang, L. Jiang, J.-M. Koo, J.G. Maveety, E.A. Sanchez, K.E. Goodson, T.W. Kenny, Micromachined jets for liquid impingement cooling of VLSI chips, J. MEMS 13 (5) (2004) 833-842.

[6] S.G. Kandlikar, Heat transfer mechanisms during flow boiling in microchannels, J. Heat Trans. 126 (2004) 8-16.

[7] R. Srikar, T. Gambaryan-Roisman, C. Steffes, P. Stephan, C. Tropea, A.L. Yarin, Nanofiber coating of surfaces for intensification of drop or spray impact cooling, Int. J. Heat Mass Transf. 52 (25-26) (2009) 5814-5826.

[8] H.S. Ahn, V. Sathyamurthi, D. Banerjee, Pool boiling experiments on a nanostructured surface, IEEE Trans. Comput. Pack. Technol. 32 (1) (2009) 156-165.

[9] V. Khanikar, I. Mudawar, T. Fishera, Effects of carbon nanotube coating on flow boiling in a micro-channel, Int. J. Heat Mass Transf. 52 (15-16) (2009) 38053817.

[10] R. Xiao, K.H. Chu, E.N. Wang, Multi-layer liquid spreading on superhydrophilic nanostructured surfaces, Appl. Phys. Lett. 94 (14) (2009) 193104.

[11] R.J. Daniello, N.E. Waterhouse, J.P. Rothstein, Drag reduction in turbulent flows over superhydrophobic surfaces, Phys. Fluids 21 (2009) 085103.

[12] C.-H. Chen, Q. Cai, C. Tsai, C.-L. Chen, G. Xiong, Y. Yu, Z. Ren, Dropwise condensation on superhydrophobic surfaces with two-tier roughness, Appl. Phys. Lett. 90 (2007) 173108.

[13] Q.F. Xu, J.N. Wang, I.H. Smith, K.D. Sanderson, Directing the transportation of a water droplet on a patterned superhydrophobic surface, Appl. Phys. Lett. 93 (2008) 2333112

[14] K. Chu, R. Xiao, E.N. Wang, Uni-directional liquid spreading on asymmetric nano-structured surfaces, Nat. Mater. (2010).

[15] P. Poesio, A.M. Lezzi, G.P. Beretta, Evidence of convective heat transfer enhancement induced by spinodal decomposition, Phys. Rev. E 75 (2007) 066306/1-066306/9.

[16] D. Molin, R. Mauri, Enhanced heat transport during phase separation of liquid binary mixtures, Phys. Fluids 19 (2007) 074102/1-074102/10.

[17] S. Gat, N. Brauner, A. Ullmann, Heat transfer enhancement via liquid-liquid phase separation, Int. J. Heat Mass Transf. 20 (2009). XX

[18] P. Poesio, G.P. Beretta, T. Thorsen, Dissolution of a liquid micro-droplet in a non-ideal liquid-liquid mixture far from thermodynamic equilibrium, Phys. Rev. Lett. 103 (6) (2009) 06450/1-06450/4.

[19] J.C. Arce, A. Schaadt, H.J. Bart, Extraction with spinodal decomposition: experiment and simulation, Chem. Eng. Technol. 29 (4) (2006) 487-494.

[20] E.P. Gyftopoulos, G.P. Beretta, Thermodynamics: Foundations and Applications, Dover Pub., 2005.

[21] S. Shen, I. Nagata, Prediction of excess enthalpies of ketone-alkane systems from infinite dilution activity coefficients, Thermochim. Acta 258 (1995) 1931.

[22] N. Vladimirova, A. Malagoli, R. Mauri, Two-dimensional model of phase segregation in liquid binary mixtures, Phys. Rev. E 60 (1999) 6968-6977.

[23] N. Vladimirova, A. Malagoli, R. Mauri, Two-dimensional model of phase segregation in liquid binary mixtures with an initial concentration gradient, Chem. Eng. Sci. 55 (2000) 6109-6118.

[24] J.M. Prausnitz, Ruediger N. Lichtenthaler, Edmundo Gomes de Azevedo, Molecular Thermodynamics of Fluid-Phase Equilibria, Prentice-Hall PTR, 1999.

[25] E. Machedo, P. Rasmussen, Liquid-liquid equilibrium data collection, DeChema Data Ser. V (part 4, 52) (1987).

[26] R.H. Perry, D.W. Green, Perry's Chemical Engineers' Handbook, McGraw-Hill Professional, 1997

[27] R.B. Bird, W.E. Stewart, E.N. Lightfoot, Transport Phenomena, Wiley, 2002.

[28] P. Poesio, G. Cominardi, A.M. Lezzi, R. Mauri, G.P. Beretta, Effects of quenching rate and viscosity on spinodal decomposition, Phys. Rev. E 74 (2006) 011507/ $1-011507 / 13$ 